\title{
The Application of combination of External Drainage from Intestinal Cavity and Peritoneal Drainage on the Surgical Treatment for Neonates with Necrotizing Enterocolitis
}

\author{
Ji-xue Zhao, ${ }^{1,}$, Xin Fu ${ }^{2}$ \\ ${ }^{1}$ Department of Pediatric Surgery, The First Hospital of Jilin University, Changchun 130021, China \\ ${ }^{2}$ Nursing Administration Department, China-Japan Union Hospital of Jilin University, Changchun \\ 130033, China \\ ${ }^{2}$ Corresponding author e-mail: jixuezhao0431@126.com
}

Keywords: Peritoneal drainage, Neonates, Necrotizing enterocolitis.

\begin{abstract}
Object: To evaluate the efficacy of combination of external drainage from intestinal cavity and peritoneal drainage on the surgical treatment for neonates with necrotizing enterocolitis(NEC). Methods: A retrospective analysis was conduct on 48 cases of newborns with extensive and multiple intestinal necrosis found intraoperatively, all of which could not accept firststage resected, treated by traditional operation, external drainage from intestinal cavity, peritoneal drainage and combination of external drainage from intestinal cavity and peritoneal drainage, between January, 2010 to January 2017. Duration of operation, amount of bleeding, and the survival rate after operation were compared among the four groups, which were divided by surgical methods. Results: No significant difference was observed in age, and gender $(\mathrm{P}>0.05)$ among the four groups. However, the duration of operation was significantly shorter, the amount of bleeding was significantly lessen, the survival rate was significantly higher in the patients of group B(external drainage from intestinal cavity), group $\mathrm{C}$ (peritoneal drainage ) and group $\mathrm{D}$ (combination of external drainage from intestinal cavity and peritoneal drainage) than the patients of group A(traditional operation $)(\mathrm{P}<0.05)$. While the survival rate of group $\mathrm{D}$ was significantly higher than that of other groups. Conclusion: The combination of external drainage from intestinal cavity and peritoneal drainage can improve the survival rate of the NEC children more obviously than any other single methods.
\end{abstract}

\section{Introduction}

Necrotizing enterocolitis (NEC) is one of the most common gastrointestinal tract disease frequently affecting premature infant with an incidence of $3 \%$ to $5 \%$, depending on the gestational age (GA) of infant at birth,[1,2] and mortality rate of as high as 50\%.[3,4] Although most cases of NEC are managed conservatively, with optimum medical treatment, bowel rest, abdominal decompression, parenteral nutrition, as well as antibiotics treatment, surgical consultation should be done at the beginning of NEC treatment.[5] Emergency surgical intervention is considered in condition with deteriorating clinical condition and intestinal perforation.[6,7]

The operation method for the NEC cases is to according to the degree and scope of bowel necrosis. In most situations, we resect the necrotic bowel and perform the jejunostomy, and 3 months later, ostomy closure will be performed. In the condition that the intestine was affected extensively unable to be resected on the first-stage operation, there are not a definite surgery method which is strongly recommended and agreed. The conventional surgical method is jejunostomy. But surgical time is too long and mortality was relatively high of this method. In view of this kind of situation, we adopted three new methods (external drainage from intestinal cavity, peritoneal drainage, combination of external drainage from intestinal cavity and peritoneal drainage) to replace the conditional jejunostomy in the first stage operation from January of 2010, and achived ideal clinical results. 


\section{Patients and Methods}

\subsection{Patients}

From January of 2010 to January of 2017, 48 surgically treated neonates with necrotizing enterocolitis in the neonatology department, the First Hospital of Jilin University were enrolled in this retrospective analysis. Each patient signed an informed consent form. Approval was obtained from the institutional review committee of Jilin University.

\subsection{Study Design}

The patients were divided into four groups according to the surgical methods: Group A: 12 patients were treated by traditional method, that is jejunostomy, these patients would accept surgery again 24 hours later to determine whether affected intestine segment could be reserved; Group B: 11 patients accepted external drainage from intestinal cavity; Group C: 12 patients accepted peritoneal drainage; Group D: 13 patients accepted combination of external drainage from intestinal cavity and peritoneal drainage. And for the patients of Group B,C and D, the later exploratory operation would be conducted to determine whether anastomosis or jejunostomy.

\subsection{Methods}

Traditional methods: Jejunostomy was applied in the first stage in critically affected neonates with NEC, then further operation proceeded 24 hours later to check the severity and length of affected intestine. Necrotic bowel would be resected, and jejunostomy was adopted followed anastomosis after 2 to 3 months. Improved surgery method: In the condition of extensive necrotic small intestine and colon that could not be resected in the first stage, we performed three methods: external drainage from intestinal cavity, peritoneal drainage, combination of external drainage from intestinal cavity and peritoneal drainage. 7 to 10 days later when the affected newborn's condition was stable, an exploratory surgery could be conducted to determine further surgery method. Primary enteroanastomosis was made when the affected bowel color changed to normal, or Jejunostomy was adopted when affected intestine was unsatisfying followed by enteroanastomosis two or three months later.

\subsection{Evaluation Criteria of Treatment Effects}

Evaluation criteria included the duration of operation and the survival rate after operation.

\subsection{Statistical Analysis}

All measured parameters including the duration of operation and the survival rate after operation, were analyzed by the statistical software program Statistical Product and Service Solutions (SPSS) 17.0 (SPSS Inc., Chicago, IL, USA) and expressed as mean \pm standard deviation ( \pm s), and t-test was used. Enumeration data including gender, prevalence frequency were analyzed by $\chi 2$ test. $\mathrm{P}<0.05$ was considered significant.

\section{Results}

\subsection{Baseline Characteristics}

There were no significant difference $(\mathrm{P}>0.05)$ in general data including operation age, gender among the four groups (Table 1.). 
Table 1. Baseline characteristics of study patients

\begin{tabular}{lccc}
\hline & Female / Male & Operation age (days) & $\begin{array}{c}\text { Neonatal } \\
\text { weight(kg) }\end{array}$ \\
\hline Group A & $4 / 8$ & $13.1 \pm 4.1$ & $2.5 \pm 0.8$ \\
Group B & $4 / 7$ & $12.5 \pm 3.5$ & $2.3 \pm 0.9$ \\
Group C & $3 / 9$ & $15.2 \pm 3.3$ & $2.6 \pm 0.5$ \\
Group D & $4 / 9$ & $14.1 \pm 4.5$ & $2.5 \pm 0.6$ \\
\hline
\end{tabular}

$\mathrm{P}>0.05$, compared among group A,B,C,D.

\subsection{Evaluation of Treatment Effects}

The duration of operation was significantly shorter, the amount of bleeding was significantly lessen, the survival rate was significantly higher in the patients of group B(external drainage from intestinal cavity), group $\mathrm{C}$ (peritoneal drainage ) and group $\mathrm{D}$ (combination of external drainage from intestinal cavity and peritoneal drainage) than the patients of group $\mathrm{A}($ traditional operation $)(\mathrm{P}<0.05)$. While the survival rate of group $\mathrm{D}$ was significantly higher than that of other groups $(\mathrm{P}<0.05)$. Postoperative follow-up was conducted for 6 months.

Table 2 Efficacy of the four groups

\begin{tabular}{lcccc}
\hline & $\begin{array}{c}\text { Number of } \\
\text { cases }\end{array}$ & $\begin{array}{c}\text { Duration of } \\
\text { operation } \\
\text { (minutes) }\end{array}$ & $\begin{array}{c}\text { Amount of } \\
\text { bleeding }(\mathrm{ml})\end{array}$ & survival rate \\
\hline Group A & 12 & $48.5 \pm 7.9$ & $6.3 \pm 2.4$ & $3 / 12$ \\
Group B & 11 & $25.4 \pm 4.5 \#$ & $2.9 \pm 1.5 \#$ & $6 / 11 * \triangle$ \\
Group C & 12 & $27.2 \pm 3.5 \#$ & $2.8 \pm 1.7 \#$ & $7 / 12 * \triangle$ \\
Group D & 13 & $28.3 \pm 5.1 \#$ & $3.1 \pm 1.1 \#$ & $11 / 13$ \\
\hline
\end{tabular}

* $\mathrm{P}<0.05$, compared with group $\mathrm{A} ; \triangle \mathrm{P}<0.05$, compared with group $\mathrm{D}$

\# $\mathrm{P}<0.05$, compared with group A; \# $\mathrm{P}>0.05$, compared among group $\mathrm{B}, \mathrm{C}, \mathrm{D}$.

\section{Discussion}

There were no significant difference $(\mathrm{P}>0.05)$ in general data including operation age, gender among the four groups (Table 1.). Necrotizing enterocolitis (NEC) remains the most common cause of gastrointestinal-associated morbidity and mortality in neonatal intensive care unit (NICU). Prematurity and low birth weight (LBW) are the most consistently agreed risk factors[8] .The estimated number of live newborns in China is about 16 million per year, with $26.2 \%$ preterm (gestational age $<37$ weeks) and $23.6 \%$ with LBW $(<2500 \mathrm{~g})$ [9]. NEC is a disorder characterized by ischemic necrosis of the intestinal mucosa, which is associated with inflammation, invasion of enteric gas forming organisms, and dissection of gas into the muscularis and portal venous system [10]. Early stage of NEC mostly could be cured by supportive treatment and antibiotics. Other signs that indicate bowel necrosis must be considered, including unremitting clinical deterioration [11]. However, to critically affected infants who are sensitive to medical treatment, surgical intervention was recommended.

And the best operative opportunity is the point when the intestinal wall was necrotized completely without intestinal perforation because this time necrotized bowel could be resected and lessen the intra-abdominal infection. During operation, every effort should be made to preserve as much intestinal length as possible. The option of surgical procedures largely depends upon the extent of disease and the attending surgeon's experience. The surgery method of NEC in neonates mainly depends on the severity and length of necrotic intestine [12]. In the condition that the intestine was affected extensively unable to be resected on the first-stage operation, there are not a definite surgery method which is strongly recommended and agreed. 
The conventional surgical method is jejunostomy. But surgical time is too long and mortality was relatively high of this method. Aim at this situation, we adopted three new methods (external drainage from intestinal cavity, peritoneal drainage, combination of external drainage from intestinal cavity and peritoneal drainage) to replace the conditional jejunostomy in the first stage operation.

In the condition of extensive necrotic small intestine and colon that could not be resected in the first stage, we perform the above three methods respectively in different groups. 7 to 10 days later when the affected newborn's condition was stable, an exploratory surgery could be conducted to determine further surgery method. Primary enteroanastomosis was made when the affected bowel color changed to normal, or Jejunostomy was adopted when affected intestine was unsatisfying followed by enteroanastomosis two or three months later.

And these three types of surgical methods achieved satisfactory clinical effects. The duration of operation was significantly shorter, the amount of bleeding was significantly lessen, the survival rate was significantly higher in the patients of group $\mathrm{B}$ (external drainage from intestinal cavity), group $\mathrm{C}$ (peritoneal drainage ) and group $\mathrm{D}$ (combination of external drainage from intestinal cavity and peritoneal drainage) than the patients of group $\mathrm{A}$ (traditional operation $)(\mathrm{P}<0.05)$. While the survival rate of group D was significantly higher than that of other groups. That means the combination of external drainage from intestinal cavity and peritoneal drainage can improve the survival rate of the NEC children more obviously than any other single methods.

External drainage from intestinal cavity could apparently lesson the tension of bowel wall and reduce the absorption of inflammatory mediators. Abdominal washing and peritoneal drainage could clean the abdominal cavity effectively and apparently reduce the absorption of inflammatory mediators. A reasonable mechanism of NEC is that intestinal mucosa of affected intestine was destroyed, and amounts of inflammatory mediators were generated and absorbed by bowel wall, leading to necrotized intestine, perforation and even multiple organ failure[13]. Therefore, a critical aspect of managing the patients with NEC is to reduce the absorption of inflammatory mediators.

The combination of external drainage from intestinal cavity and peritoneal drainage could apparently reduce the absorption of inflammatory mediators for the serious NEC patients, so this method is feasible and worthy to be popularized in clinic.

\section{Conclusion}

The combination of external drainage from intestinal cavity and peritoneal drainage, as an easy and feasible method for the serious NEC patients improved by us, has a satisfactory clinical effect with a relatively higher survival rate. And it should be recommended.

\section{References}

[1] Houben CH, Chan KW, Mou JW, et al. Management of intestinal strictures post conservative treatment of necrotizing enterocolitis: the long term outcome. J Neonatal Surg 2016;5:28.

[2] Heida FH, Loos MH, Stolwijk L, et al. Risk factors associated with postnecrotizing enterocolitis strictures in infants. J Pediatr Surg 2016;51:1126-30.

[3] Moss RL, Kalish LA, Duggan C, et al. Clinical parameters do not adequately predict outcome in necrotizing enterocolitis: a multiinstitutional study. J Perinatol 2008;28:665-74.

[4] Holman RC, Stoll BJ, Curns AT, et al. Necrotising enterocolitis hospitalisations among neonates in the United States. Paediatr Perinat Epidemiol 2006;20:498-506.

[5] Kliegman RM, Stanton BF, Geme III JW , Schor NF, Behrman RE. Nelson Textbook of Pediatrics. 19th ed.WB Saunders, Philadelphia, PA:2011;108-12.

[6] Gaudin A, Farnoux C, Bonnard A, et al. Necrotizing enterocolitis (NEC) and the risk of intestinal stricture: the value of C-reactive protein. PLoS One 2013;8:e76858. 
[7] Muensterer OJ, Keijzer R. Single-incision pediatric endosurgery-assisted ileocecectomy for resection of a NEC stricture. Pediatr Surg Int 2011;27:1351-3.

[8] Henry MC, Moss RL. Necrotizing enterocolitis. Annu Rev Med 2009;60:111-24.

[9] Subspecialty Group of Neonatology, Pediatric Society, Chinese Medical Association.Epidemiologic survey for hospitalized neonates in China. Zhongguo Dang Dai Er Ke Za Zhi 2009;11:15-20. [Article in Chinese].

[10] Neu J. Necrotizing enterocolitis: the search for a unifying pathogenic theory leading to prevention. Pediatr Clin North Am 1996; 43:409.

[11] Tepas JJ 3rd, Sharma R, Leaphart CL, et al. Timing of surgical intervention in necrotizing enterocolitis can be determined by trajectory of metabolic derangement. J Pediatr Surg 2010; 45:310.

[12] Qinmiao Lin, Yongbiao Wang, ZR Li.Surgical treatment on necrotizing enterocolitis in newborns [J]. Journal of Applied Clinical Pediatrics. 2010, 6 (11) 814-816.

[13] HY Wu, Y Yang. A analysis on risk factors in necrotizing enterocolitis in newborns. Chinese And Foreign Medical Research.2013,11(32):38-39. 